\title{
Cue dependent right hemineglect in schizophrenia: a kinematic analysis
} Michelle E Downing, James G Phillips, John L Bradshaw, Krishna S Vaddadi,
Christos Pantelis
Department of

Psychology, Monash

University, Clayton

VIC 3168, Australia

M E Downing

J G Phillips

J L Bradshaw

Adult Psychiatry, Monash Medical

Centre, Clayton VIC

3168, Australia

K S Vaddadi

Cognitive

Neuropsychiatry Unit,

Mental Health

Research Institute, and

Department of

Psychiatry, University

of Melbourne,

Parkville VIC 3052,

Australia

C Pantelis

Correspondence to:

Dr J G Phillips, Psychology

Department, Monash

University, Clayton VIC

3168, Australia. Fax 00613

99053948.

Received 9 April 1997 and in final revised form

15 December 1997

Accepted 14 January 1998

\begin{abstract}
Objectives-Schizophrenia may result from disturbed attentional processes and/or defective internal cueing. Attention for subsequent action within a cued movement task was therefore studied, testing specific hypotheses of hemispheric dysfunction and of impaired interhemispheric communication.

Method-Fifteen patients with schizophrenia and 15 matched controls were either cued or uncued when moving a pen to a target on their right or left side with their right or left hand. Pen tip position was sampled at $200 \mathrm{~Hz}$ on a WACOM SD420 graphics tablet for subsequent kinematic analysis.

Results-Patients with schizophrenia were slower initiating rightwards movements without a cue. Patients also exhibited reduced abductive/adductive differences in the shape of their movement trajectories, implying differences in interhemispheric communication.

Conclusions-It is speculated that schizophrenia is a form of cue dependent right hemineglect.
\end{abstract}

(F Neurol Neurosurg Psychiatry 1998;65:454-459)

Keywords: schizophrenia; hemineglect; attention; programming; kinematics

Patients with schizophrenia may have left hemispheric dysfunction, according to anomalous structural asymmetries seen in some brain imaging. ${ }^{12}$ Indeed patients with schizophrenia exhibit greater right sided parkinsonism, ${ }^{3}$ anomalous left directional turning biases, ${ }^{4}$ and behaviour suggestive of right hemineglect ${ }^{5}$ (implying left hemispheric dysfunction). Any right neglect ${ }^{67}$ may reflect lateralised impairment in attentional processes. We therefore considered whether schizophrenia leads to the exhibition of a neglect-like pattern of attentional deficits associated with the right hemispace.

Studies considering the direction of attention in schizophrenia are unclear. Wheras several researchers have found that patients experience difficulty shifting visual attention to the right visual field, ${ }^{89}$ others report difficulty shifting attention to the left visual field, ${ }^{10}$ and others have found no difference between leftwards and rightwards attentional shifts. ${ }^{11}{ }^{12}$ However, these studies have used external cues, whereas patients with schizophrenia are typically thought to have problems with internal cues. $^{13}$ We therefore considered whether patients with schizophrenia exhibit lateralised deficits in the direction of their attention associated with internally and externally cued actions.

Neglect has motor as well as sensory features. ${ }^{14} 15$ We therefore investigated whether patients with schizophrenia exhibit disproportionate problems in the initiation or the execution of cued and uncued movements into the left or right hemispace (hemispheric dysfunction). Definite difficulties associated with a specific hemispace (indicated by group by hemispace interactions) would imply dysfunction of the opposite hemisphere. Nevertheless, it is possible that patients merely have disproportionate problems executing movements into the opposite hemispace (indicated by group by hand by hemispace interactions), which instead would imply impaired interhemispheric communication. ${ }^{16}$

\section{Patients and methods}

PARTICIPANTS

Fifteen patients diagnosed with schizophrenia according to DSM-IV criteria, with a mean age of 30.07 (SD 7.14) years, were matched for age and sex with 15 healthy normal controls (mean age 29.67 (SD 7.39) years). All participants were right handed (as determined by a handedness questionnaire ${ }^{17}$ ), and gave informed consent for these procedures under the guidelines of each hospital's ethics committee. Eight patients were on atypical neuroleptic drugs (for example, clozapine), and seven patients were on more typical neuroleptic drugs (for example, chlorpromazine, haloperidol). The table shows the patients' characteristics.

All participants were screened for uncorrected visual impairment; previous head injury, neurological or motor impairment; limb injury or arthritis; history of epilepsy; and recent recreational drug use. Patients with a history of other psychiatric illnesses in addition to schizophrenia were also excluded. The presence of involuntary movements was assessed using the abnormal involuntary movement scale $\left(\mathrm{AIMS}^{18}\right.$ ) and in some cases also by the consulting psychiatrist. Only those patients exhibiting minimal or no involuntary movement dysfunction were included in the patient sample. Controls were drawn from the community by personal appeal and were screened for known history of schizophrenia or other serious psychiatric illness; familial psychiatric disturbances; and a history of neuroleptic treatment. 


\begin{tabular}{|c|c|c|c|c|c|}
\hline \multirow[b]{2}{*}{ Sex } & \multirow[b]{2}{*}{ Age } & \multirow[b]{2}{*}{ Illness duration $(y)$} & \multirow[b]{2}{*}{ Medication regime at time of testing } & \multicolumn{2}{|c|}{ Typical neuroleptics } \\
\hline & & & & Exposure $(y)$ & Last taken (months) \\
\hline $\mathrm{F}$ & 33 & 11 & Clozapine $(100 \mathrm{mg} /$ day $)$ & 10 & 9 \\
\hline M & 23 & 3 & Clozapine (175 mg/am; 200 mg/pm) & 3 & 8 \\
\hline M & 32 & 12 & Clozapine $(300 \mathrm{mg} / \mathrm{am} ; 400 \mathrm{mg} / \mathrm{pm})$ & 6 & 25 \\
\hline M & 33 & 16 & Clozapine ( $200 \mathrm{mg} / \mathrm{am} ; 400 \mathrm{mg} / \mathrm{pm})$, omeprazole $(20 \mathrm{mg} \mathrm{bd})$ & 15 & 16 \\
\hline M & 37 & 7 & Clozapine ( $900 \mathrm{mg} /$ day), clonazepam ( $0.5 \mathrm{mg} /$ day $)$ & 5 & 21 \\
\hline M & 23 & 8 & Clozapine $(500 \mathrm{mg} / \mathrm{day})$ & 8 & 6 \\
\hline M & 29 & 9 & Clozapine (200 mg/am; $300 \mathrm{mg} / \mathrm{pm})$ & 8 & 12 \\
\hline M & 40 & 28 & Clozapine $(500 \mathrm{mg} / \mathrm{day})$ & 12 & 2 \\
\hline M & 18 & 0.17 & $\begin{array}{l}\text { Chlorpromazine } \mathrm{HCl}(100, \mathrm{tid}) \text {, flupenthixol decanoate }(60 \mathrm{mg} \text { im } \\
2 / 52) \text {, sertraline } \mathrm{HCl}(150 \mathrm{mg} / \mathrm{am}) \text {, lorazepam }(0.5 \mathrm{mg} \mathrm{bd})\end{array}$ & 0.17 & * \\
\hline $\mathrm{F}$ & 24 & 3 & $\begin{array}{l}\text { Chlorpromazine }(100 \mathrm{mg}, \mathrm{prn}) \text {, benztropine mesylate ( } 1 \mathrm{mg} \mathrm{bd}) \text {, } \\
\text { thiothixene }(10 \mathrm{mg} \mathrm{am})\end{array}$ & 4 & * \\
\hline M & 36 & 11 & $\begin{array}{l}\text { Haloperidol ( } 10 \mathrm{mg} \text {, tid), benztropine mesylate }(1 \mathrm{mg} \text { bd }) \\
\text { zuclopenthixol decanoate }(250 \mathrm{mg} \text { im } 2 / 52)\end{array}$ & 11 & * \\
\hline M & 21 & 1 & Risperidone $(2 \mathrm{mg} /$ day $)$ & 1 & * \\
\hline M & 41 & 15 & $\begin{array}{l}\text { Thiothixene ( } 30 \mathrm{mg} / \text { day), buspirone } \mathrm{HCl}(175 \mathrm{mg} / \text { day }) \text {, doxepin } \mathrm{HCl} \\
\text { (10 mg tid) }\end{array}$ & NA & * \\
\hline $\mathrm{F}$ & 27 & 2 & Trifluoperazine $(20 \mathrm{mg})$ & 2 & * \\
\hline $\mathrm{F}$ & 34 & 5 & Risperidone ( $5 \mathrm{mg} \mathrm{bd})$, fluoxetine $\mathrm{HCl}(20 \mathrm{mg} /$ day $)$ & $>5$ & * \\
\hline
\end{tabular}

*Typical neuroleptic drugs taken on day of testing. $\mathrm{NA}=$ not available. $\mathrm{Bd}=$ twice daily; tid=thrice daily; prn=as required; im2/52=intramuscularly every two weeks.

APPARATUS AND TASK

In a simplified version of a previously used task, ${ }^{19}$ participants moved a non-inking pen leftwards (into the right hemispace) or rightwards (into the left hemispace), with right or left hand to connect two circular targets on a graphics tablet (WACOM SD420) in response to the illumination of light emitting diodes. The graphics tablet was situated in the midline, about $100 \mathrm{~mm}$ in front of the participant, and the $1.5 \mathrm{~cm}$ diameter targets were situated $8 \mathrm{~cm}$ from the left or right of the midline. The graphics tablet was connected to a laptop computer (MITAC 486), which sampled the position in $x$ and $y$ coordinates of the non-inking pen (accurate to $+/-0.2 \mathrm{~mm}$ ) at 200 $\mathrm{Hz}$ (every $5 \mathrm{~ms}$ ). To reduce friction, a clear plastic sheet was fixed to the writing surface of the tablet.

The illumination of the light emitting diodes was governed by a computer program and allowed manipulation of the amount of cueing available to direct movements. ${ }^{19}$ Although movements were always made across to the target in the opposite hemispace, a light emitting diode was either illuminated (cue condition) or not illuminated (no cue condition) before initiation of a movement.

LEVELS OF CUEING

Cue

Light emitting diode illumination preceded movement initiation - that is, participants were provided with an external cue for movement, which could assist the programming of movement.

No cue

Not until the pen had left the target would a light emitting diode on the opposite side illuminate - that is, participants had to generate their own internal cue for movement.

PROCEDURE

There were four blocks of trials which varied advance information (cue, no cue) and hand (left, right), their order of presentation being determined by a latin square. ${ }^{20}$ Each block consisted of 16 trials, in which participants alternately started on the left or right target (indicating a rightwards or leftwards movement), to produce the eight rightwards and eight leftwards trials for each cell of the advance information by hand by hemispace $(2 \times 2 \times 2)$ design. For each block of trials, the experimenter indicated the hand (left or right) to be used, and demonstrated the task. Participants then practised to a level where they understood, and could perform the task without any further prompting. The experimenter indicated the starting target (left side or right side) before each trial, and the starting target was also indicated by a light emitting diode. The computer bleeped when the pen entered a target. Participants were instructed to aim for the centre of the target, and to perform the drawing movement as quickly and accurately as possible. Dependent variables were submitted to separate $2 \times 2 \times 2 \times 2$ mixed model analysis of variance to compare between group (patients, controls) with repeated measures on advance information (cue, no cue), hand (left, right) and hemispace (left, right).

PROGRAMMING

Pause time (PT) was employed as an index of preparatory processes, and was the time (ms) spent stationary at a target before movement to the next target was initiated. PT reflects the time required to identify the imperative stimulus (in the cue condition), and to programme or plan the movement (in the cue and no cue conditions).

\section{REPROGRAMMING}

The following measures document the extent to which participants were required to make on line corrections to movement during its execution.

Movement time (MT) is the total time (ms) spent moving between consecutive targets in each trial, excluding the time spent pausing at targets.

Force inefficiency (FI) index reflects the number of accelerative and decelerative impulses per submovement and as such is a measure of the efficiency of movement execution. ${ }^{21}$ Submovements were determined 


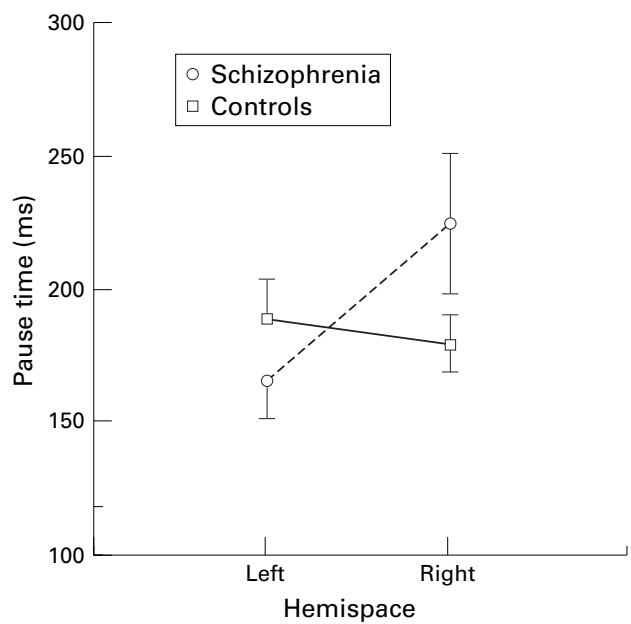

Figure 1 Mean pause time (ms) for patients with schizophrenia and their controls, when initiating movements into the left and right hemispace.

to occur between zero crossings in the velocity function. The FI index is calculated by dividing the number of zero crossings in the acceleration function by the number of zero crossings in the velocity function. An efficient movement should only have one cycle of acceleration and deceleration, while an index greater than one indicates additional cycles of acceleration and deceleration, and an inefficient movement.

The asymmetry ratio (AR) measures the shape of the movement trajectory, and is the proportion of time spent in the accelerative phase of the movement relative to the time spent in the decelerative phase of the movement. The AR is calculated by dividing the time to peak velocity by the total movement duration. An efficient movement should have accelerative and decelerative phases of approximately equal duration. A ratio $>0.5$ indicates prolonged acceleration, whereas a ratio $<0.5$ indicates prolonged deceleration or terminal guidance. ${ }^{22}$ The submovement with the greatest velocity (the primary movement) within a trial was selected for this analysis, as it was assumed that this would be the programmed (or reprogrammed) movement. ${ }^{19}$

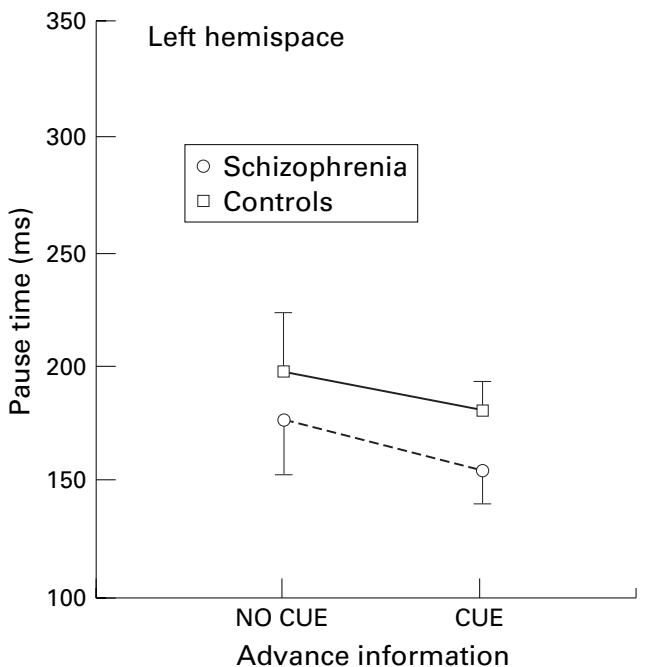

Constant error was used to indicate the accuracy of the termination of the movements in relation to the centre of the target. Constant error was calculated from the displacement data, using the difference between the stroke end point and the target centre.

\section{Results}

We only report those effects directly related to the hypothesis of hemispheric dysfunctions in attention (pause time data and group by hemispace interactions), and that of impaired interhemispheric communication (group by hand by hemispace interactions) in schizophrenia.

PROGRAMMING

The groups did not differ in amount of time required to initiate their movements, $F(1,28)=0.090, \mathrm{p}>0.05$. Evidence of hemispheric dysfunctions in the allocation of attention was provided by a significant group by hemispace interaction $(F(1,28)=4.443$, $\mathrm{p}<0.05$, fig 1). Patients seem to be slower initiating movements into the right hemispace, but this effect is cue dependent, as there was a significant three way group by hemispace by advance information interaction, $F(1,28)=8.853, \mathrm{p}<0.01$.

Figure 2 indicates that the significant three way interaction between group, hemispace and advance information, was driven by patients being slower initiating movements in a rightwards direction, in the absence of an external cue. This three way interaction was broken down into two component two way interactions, one for rightwards movements (into the right hemispace) and one for leftwards movements (into the left hemispace). A two way analysis of variance (ANOVA) disclosing that when movements were directed into the right hemispace, patients' and controls' PTs were found to significantly interact as a function of the level of advance information, $F(1,28)=7.283, \mathrm{p}<0.05$. Conversely, when movements were directed into the left hemispace, patients' and controls' PTs were not found to differ from one another as a function

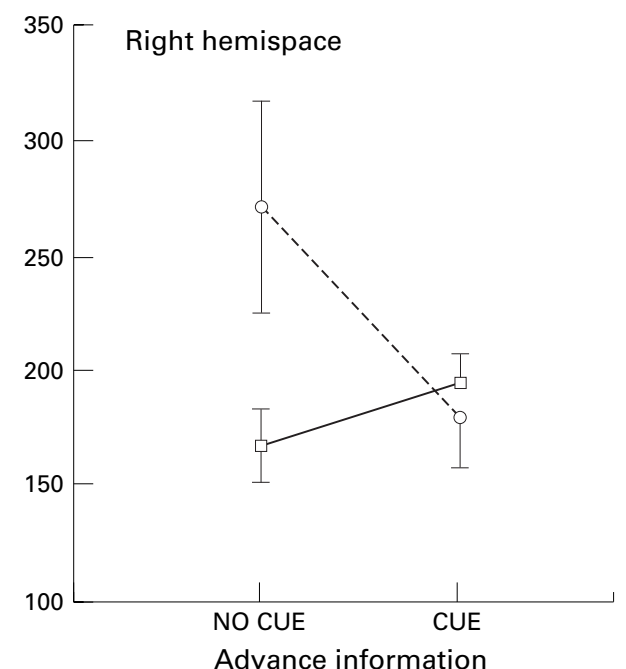

Figure 2 Mean pause time (ms) for patients with schizophrenia and their controls, when intiating cued or uncued movements into the left or right hemispace. 

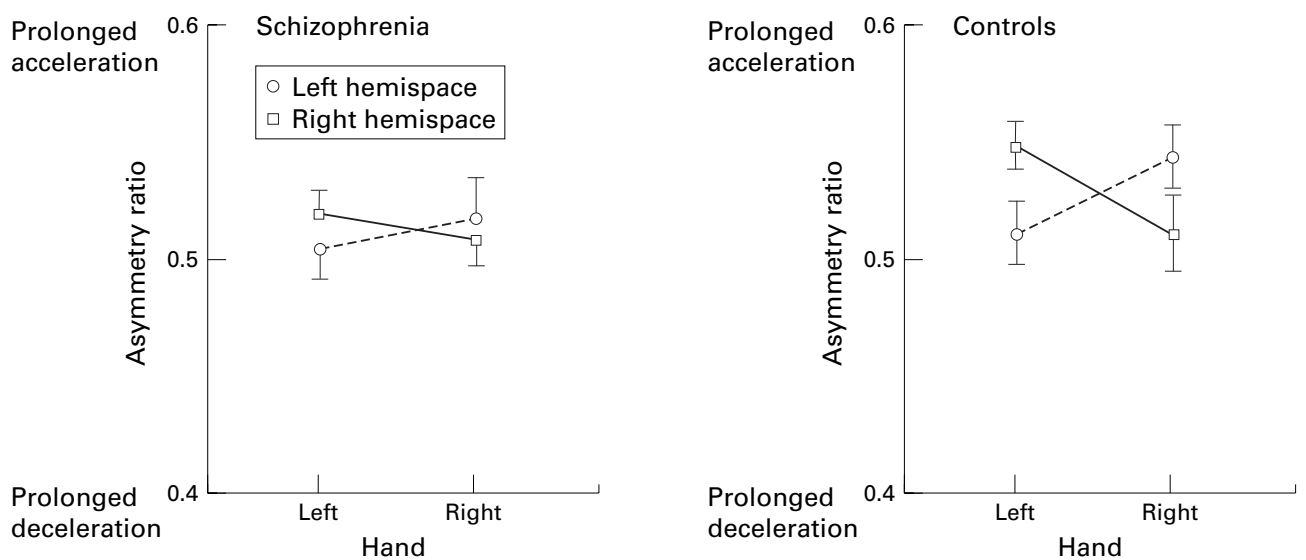

Figure 3 Relative proportion of time spent in accelerative or decelerative phases of movement, when patients move with left or right hand, into left or right hemispace.

of the level of advance information $(F(1,28)=0.035, \mathrm{p}>0.05)$. Whereas this supports a hypothesis of hemispheric dysfunction, patterns of interhemispheric communication were relatively normal during movement initiation, as the group by hand by hemispace interaction was not significant $(F(1,28)=2.922$, $\mathrm{p}>0.05$ )

Right hemispatial neglect in schizophrenia has been reported to be a function of medication status. ${ }^{11}$ The patients on atypical neuroleptic drugs (particularly clozapine) were therefore of interest, as atypical neuroleptic drugs are less likely to have side effects on movement (having a lower affinity for $\mathrm{D}_{2}$ receptors). ${ }^{23}$ The cue dependent problems initiating rightwards movements (fig 2), would not seem to be a simple function of $\mathrm{D}_{2}$ receptor blockade as this aforementioned three way interaction involving group, advance information, and hemispace, was also seen in the subset of patients on clozapine $(F(1,14)=9.806, \mathrm{p}<0.01)$.

REPROGRAMMING

Movement time (MT)

There was some evidence of bradykinesia, with patients (MT $=801.82 \mathrm{~ms}$ ) taking significantly longer to perform their movement than controls $\quad(\mathrm{MT}=597.03 \mathrm{~ms} ; \quad F(1,28)=20.651$, $\mathrm{p}<0.001)$. However, the overall MT of patients did not differ from controls as a function of the hemispace into which movements were directed $(F(1,28)=1.351, \mathrm{p}>0.05)$. Nor was there a group by hand by hemispace interaction $(F(1,28)=0.580, \mathrm{p}>0.05)$. Together, these results imply the absence of any hemispheric dysfunction at least with respect to movement directed into either hemispace, and an absence of anomalies in interhemispheric communication. Nevertheless, a kinematic analysis allows a better understanding of factors contributing to the longer MT of patients.

\section{Force inefficiency}

There was little indication that the groups varied in the numbers of submovements they made. However, schizophrenic patients $(\mathrm{M}=3.56)$ displayed an increased number of accelerative/decelerative impulses per sub- movement compared with controls $(M=2.85$; $F(1,28)=9.483, \mathrm{p}<0.01)$. The absence of interactions implied no anomalies of interhemispheric communication as far as movement efficiency was concerned.

\section{Asymmetry ratio}

Patients and controls spent about the same proportions of time in the accelerative and decelerative phases of the primary movement, as indicated by their respective mean asymmetry ratios of 0.513 and $0.529(F(1,28)=0.747$, $\mathrm{p}>0.05)$. In addition, both tended to spend relatively more time in the accelerative phase of the movement, as denoted by ratios $>0.5$.

Patients and controls spent about the same proportion of time in acceleration and deceleration when moving into the right and left hemispace. A significant group by hand by hemispace interaction suggested that there may be subtle anomalies of interhemispheric communication $(F(1,28)=5.431, \mathrm{p}<0.05$, fig 3$)$. When controls made abductive movements into ipsilateral hemispace (either when the left hand moved into the left hemispace, or the right hand moved into the right hemispace) the two hands spent about the same proportion of time in the accelerative and decelerative phases. Conversely, when the hand performing the task was required to move adductively across and into the opposite hemispace, prolonged periods of acceleration occurred, perhaps reflecting increased planning time. ${ }^{19}$ Patients with schizophrenia behaved differently when performing such movements, spending about the same proportion of time in acceleration and deceleration irrespective of whether movements were made into the ipsilateral (abductive move) or contralateral (adductive move) hemispace in relation to the hand performing the task. Whereas the same general pattern of results were seen in the clozapine patient subgroup, the group by hand by hemispace interaction was not significant, $(F(1,14)=2.045, \mathrm{p}>0.05)$ implying that medication may play a part here.

\section{Constant error}

No significant group differences were found indicating that patients' and controls' drawing 
strokes were equally accurate $(F(1,28)=2.952$, $\mathrm{p}>0.05)$.

\section{Discussion}

The present study considered the allocation and shifting of attention for action in patients with schizophrenia, considering whether schizophrenia caused hemispheric dysfunction or deficits in interhemispheric communication. There were indications that schizophrenic patients had greater difficulty initiating movements into the right hemispace when no cue was provided to trigger movement, and some suggestions of altered interhemispheric communication when executing movements into the opposite hemispace.

\section{HEMISPHERIC DYSFUNCTION}

The measure of primary interest in investigating shifts in the allocation of attention is pause time. The results suggest a lateralised deficit, with schizophrenic patients taking significantly longer to self initiate movements into the right hemispace without the aid of a cue to trigger movement. This finding may in part explain the inconsistent reports of rightwards attentional deficits in schizophrenia. Posner et at found that schizophrenic patients experienced great difficulty shifting covert visual attention to the right visual field to identify a target, after invalid precuing had indicated that the target might appear in the left visual field. In the present study, similar effects were found for self initiated movements, in which patients had to deliberately disengage attention from an external cue presented in the left hemispace, so as to prepare and initiate an internally driven voluntary movement towards an uncued target presented in the right hemispace. Any previous inconsistencies in the literature may reflect differences in the types of tasks used, as deficits emerge with self initiated behaviours. Indeed, the cue dependent nature of this deficit is reminiscent of basal ganglia dysfunction. ${ }^{24}$ However, whereas the schizophrenic patients clearly demonstrated a rightwards attentional deficit, this seemed not to be associated with an explicit rightwards motor deficit.

The impaired ability to direct attention to the right hemispace, seen in the current experiment, is also seen in patients with right hemineglect due to left sided stroke. ${ }^{25}$ In addition, hemiparkinsonian patients tend to turn towards the side of the damaged striatum. ${ }^{26}$ Similar forms of motor hemineglect have been noted in animals when unilateral striatal dopamine deficiencies have been induced. ${ }^{27}$ In such instances, rotation and attention is directed towards the hemisphere with lower striatal dopaminergic activity. Given the potential role of dopamine, it may have been preferable in the present study to examine neuroleptic naive patients with schizophrenia, but this is difficult in practice for ethical, medical, and social reasons. ${ }^{28}$ Nevertheless, the present findings of a rightwards hemi-inattention appear congruent with Bracha's ${ }^{4}$ findings of a leftwards turning preference in a group of unmedicated schizophrenic patients.
We have found long lasting effects of right hemispheric strokes, which continue to manifest as subclinical left hemineglect, ${ }^{29}$ in less explicit tasks in which internal criteria are used to make judgements. Given that right hemineglect after stroke is extremely rare, we speculate that schizophrenia is a residual subclinical hemineglect, which manifests when less explicit cues to action are available.

\section{INTERHEMISPHERIC COMMUNICATION}

There were some qualitative differences associated with movements into the opposite hemispace. Controls spent proportionately longer in the accelerative phase of movement, before movements into the opposite hemispace, than did patients with schizophrenia. Whereas controls seem to exhibit a potential handhemispace compatibility, this is less noticeable in patients with schizophrenia. This finding implies that there may be subtle differences in interhemispheric communication in patients with schizophrenia. ${ }^{30}$

\section{Conclusion}

The findings of a right sided hemi-inattention in the present experiment suggest possible abnormalities in dopamine related hemispheric asymmetries in schizophrenia. Specifically, the right sided hemi-inattention may have arisen as a consequence of relative hypofunctionality of the left, or relative hyperfunctionality of the right hemisphere, with the cue dependent nature of this deficit implying frontostriatal dysfunction.

We gratefully acknowledge the assistance of Dr David Leonard and Mornington Peninsula Hospital. This work was supported by grants from the Faculty of Science, Monash University, and the Australian Research Council. The work was performed by Michelle Downing as part of the requirements of the BSc (hons) degree.

1 Crow TJ, Brown R, Bruton CJ, et al. Lateral asymmetry of temporal horn enlargement in schizophrenia. Neurosci Lett 1988;32:S58.

2 Early TS, Reiman EM, Raichle ME, et al. Left globus pallidus abnormality in never-medicated patients with schizophrenia. Proc Natl Acad Sci US A 1987;84:561-3.

3 Caligiuri MP, Lohr JB, Jeste DV. Parkinsonism in neuroleptic-naive schizophrenic patients. Am F Psychiatry 1993;150:1343-8

4 Bracha HS. Asymmetrical rotational (circling) behavior, a dopamine-related asymmetry: preliminary findings in unmedicated and never medicated schizophrenic patients. Biol Psychiatry 1987;22:995-1003.

5 Early TS. Left globus pallidus hyperactivity and right-sided hemineglect in schizophrenia. In: Cromwell RL, Snyder $\mathrm{CR}$, eds. Schizophrenia: origins, processes, treatment and outcome. New York: Oxford University Press 1993:17-30.

6 outcome. New York: Oxford University Press 1993:17-30. attentional abnormality in schizophrenia is correlated with attentional abnormality in schizophrenia is correlat

7 Goldsamt LA. Neuropsychological findings in schizophrenia. In: Koziol LF, Stout CE, eds. The neuropsychology of mental disorders. Springfield, Illinois: Charles C Thomas, mental disorders.

8 Posner MI, Early TS, Reiman E, et al. Asymmetries in hemispheric control of attention in schizophrenia. Arch Gen Psychiatry 1988;45:814-21

9 Potkin SG, Swanson JM, Urbanchek M, et al. Lateralized deficits in covert shifts of visual attention in chronic and never-medicated schizophrenics compared to normal controls. Schizophr Res 1989;2:95.

10 Moran MJ, Thaker GK, Smith D, et al. Shifts in covert visual attention in schizophrenic patients and normal controls. Biol Psychiatry 1992;32:617-620.

11 O'Carroll RE, Rogers A, Lawrie SM, et al. Laterality of visuo-spatial attention in acute and chronic schizophrenia, major depression and in healthy controls. Psychol Med major depression

12 Strauss ME, Novakovic T, Tien AY, et al. Disengagement of attention in schizophrenia. Psychiatry Res 1991;37:139-46.

13 Frith CD. The cognitive neuropsychology of schizophrenia. Hove, Sussex: Lawrence Erlbaum, 1992 
14 Heilman, KM, Valenstein E, Watson RT. Disorders of attention. In: Trimble MR, Cummings JL, eds. Contemporary behavioral neurology. Boston: Butterworth-Heinemann, 1997:127-37.

15 Mattingley JB, Bradshaw JL, Phillips JG. Impairments of movement initiation and execution in unilateral neglect: directional hypokinesia and bradykinesia. Brain 1992;115 1849-74.

16 Morgan M, Bradshaw JL, Phillips JG, et al. Effects of hand and age upon abductive and adductive movements: a kinematic analysis. Brain Cogn 1994;25:194-206.

17 Bradshaw JL, Bradshaw JA, Nettleton NC. Abduction, adduction and hand differences in simple and serial movements. Neuropsychologia 1990;28:917-31.

18 Department of Health, Education and Welfare. Abnormal involuntary movement scale. Washington, DC: Alcohol, Drug Abuse, and Mental Mealth Administration, DHEW, 1974.

19 Bellgrove MA, Phillips JG, Bradshaw JL, et al. Response programming in dementia of the Alzheimer type: a programming in dementia of the Alzheimer type:
kinematic analysis. Neuropsychologia 1997;35:229-40.

20 Ferguson GA, Takane Y. Statistical analysis in psychology and education. New York: McGraw-Hill, 1989.

21 Hogan N, Flash T. Moving gracefully: quantitative theories Hogan N, Flash T. Moving gracefully: quantitative theorie
of motor coordination. Trend Neurosci 1987;10:170-4.

22 Nagasaki H. Asymmetric velocity and acceleration profiles Nagasaki H. Asymmetric velocity and acceleration profiles
of human arm movements. Exp Brain Res 1989;74:319-26.

23 Cunningham Owens DG. Advances in psychopharmacology—schizophrenia. Br Med Bull 1996; 52:556-74.
24 Jones DL, Phillips JG, Bradshaw JL, et al. Programming of single movements in Parkinson's disease: comparisons with Huntington's disease. F Clin Exp Neuropsychol 1992;4:76272 .

25 Early TS, Haller JW, Posner MI, et al. The left striatopallidal hyperactivity model of schizophrenia. In: David AS, Cutting JC, eds. The neuropsychology of schizophrenia. Sussex: Lawrence Erlbaum, 1994:15-38.

26 Bracha HS, Shultz C, Glick SD, et al. Spontaneous asymmetric circling behavior in hemi-Parkinsonism: a human equivalent of the lesioned-circling rodent behavior. Life Sci 1987;40:1127-30.

27 Miyashita N, Hikosaka O, Kato M. Visual hemineglect induced by unilateral striatal dopamine deficiency in monkeys. Neuroreport 1995;6:1257-60.

28 Manschreck TC, Maher BA, Rucklos ME, et al. Disturbed voluntary motor activity in schizophrenic disorders. Psychol Med 1982;12:73-84.

29 Mattingley JB, Bradshaw JL, Bradshaw JA, et al. Residual rightward attentional bias after apparent recovery from right hemisphere damage: implications for a multicomponent model of neglect. $\mathcal{F}$ Neurol Neurosurg Psychiatry 1994;57:597-604.

30 Gruzelier J. Lateralised dysfunction is necessary but not sufficient to account for neuropsychological deficits in schizophrenia. In: Pantelis C, Nelson HE, Barnes TRE, eds. Schizophrenia: a neuropsychological perspective. Chichester: John Wiley, 1996:125-60. 